\title{
Post-poliomyelitis syndrome
}

This article was published in the following Dove Press journal:

International Medical Case Reports Journal

\author{
Eric Chun Pu Chu' \\ Kary Ka Wai Lam² \\ 'New York Chiropractic and \\ Physiotherapy Center, New York Medical \\ Group, Hong Kong, People's Republic of \\ China; ${ }^{2}$ Downtown Chiropractic Limited, \\ Hong Kong, People's Republic of China
}

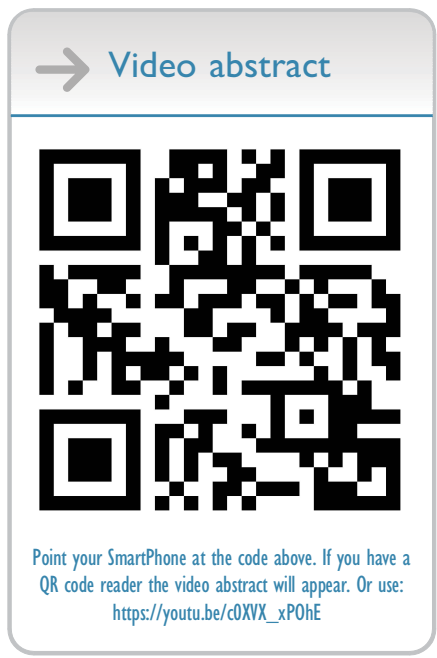

Correspondence: Eric Chun Pu Chu New York Chiropractic and Physiotherapy Centre, New York Medical Group, 4I/F Langham Place Office Tower, 8 Argyle Street, Mongkok, Hong Kong, People's Republic of China

Tel +852 35947844

Fax +85235946193

Email eric@nymg.com.hk

\begin{abstract}
Most developed countries eliminated paralytic poliomyelitis (polio) in the 1970 s to 1980 s. It was believed that after recovery from acute paralytic poliomyelitis, the physical condition of survivors would remain stable for the rest of their lives. However, the elimination of polio does not equate the end of medical management of polio. Hundreds of thousands of polio survivors worldwide are still at risk of developing the late effects of the disease. Here, we report a case of post-polio syndrome who attended our clinic for the presence of new weakness and neuromuscular problems six decades after recovery from paralytic polio. It is essential that health professionals be aware of these conditions and have an understanding of the underlying pathophysiology of the symptoms.
\end{abstract}

Keywords: late effects of polio, paralytic poliomyelitis, polio survivor, post-polio syndrome

\section{Introduction}

Poliovirus (PV) is a member of human enterovirus species C. ${ }^{1}$ All enteroviruses are quite resistant in the environment. Poliomyelitis primarily affects children under 5 years of age and spreads mainly through the fecal-oral route. Despite being highly contagious, the severity of the disease depends largely on the site of viral target. On entry of the human systems, the virus diverts the translation machinery in its favor, causing inhibition of cellular protein synthesis. ${ }^{1}$ The vast majority (90-95\%) of infections is confined to the gastrointestinal tract and people who contract PV are often asymptomatic or have mild symptoms (known as subclinical polio). Less than $1 \%$ of PV infections result in paralytic poliomyelitis (often called polio). This viral strain targets the motor neurons in the spinal anterior horn and the brain stem, leading to muscle weakness and flaccid paralysis. ${ }^{2}$ Polio has almost been eradicated from developed countries since the introduction of the Salk (1955) and Sabin (1962) polio vaccines. Acute polio is rare nowadays due to the success of poliovirus vaccine.

In the late 1970 s, however, many polio survivors, who had contracted poliomyelitis in the 1940s and 1950s, noticed clinical deterioration (such as pain, muscle weakness and muscle fatigability). ${ }^{3}$ The term "post-polio syndrome" (PPS) was coined in the $1980 \mathrm{~s},{ }^{4}$ describing this new, late manifestation of polio several decades following the initial recovery. The pathophysiology behind PPS remains unclear but is likely multifactorial. ${ }^{5}$ Deterioration of the neuromuscular functions, overuse of the motor units, the general ageing process, and inflammatory changes in the central nervous system and serum, have all been proposed as possible explanations for the new symptoms. ${ }^{6}$ Moreover, the late effects of polio (including arthritis, tendinitis, muscle wear, osteoporosis and fracture), often a consequence of biomechanical alterations, may mimic PPS or be magnified by PPS. This population deserves multidisciplinary attention. 


\section{Case report}

A 59-year-old woman presented with back pain, progressive muscle weakness and cramps in the left leg. She gave a history of paralytic polio at the age of 2, which left her with permanent flaccid weakness in the left upper limb. She stated that her left leg was also initially affected but it had subsequently recovered. The new symptoms (weakness and cramps) were mainly in the muscle groups of the left (recovered) leg, progressing for over one year. The leg weakness was accompanied by low back soreness. After consulting her orthopedic doctor, she was prescribed with ibuprofen (a nonsteroidal anti-inflammatory drug), which provided little relief.

On examination, the patient presented with a waddling gait and could walk with a cane. She had muscle wasting of the left arm and forearm, showing clear-cut evidence of old polio. The right arm and right leg were neurologically normal. Motor strength in the lower limbs was graded as 4/5 at left L3-S1, and 5/5 at right L3-S1. Except for lumbar scoliosis, the X-ray findings were essentially normal. Needle EMGs revealed denervation of the muscle groups in both the left upper and lower limbs. Surface electromyography (MyoVision $\AA$ ) revealed severe spasm of bilateral paraspinal muscles throughout the spine. She met the diagnostic criteria ${ }^{3}$ for post-poliomyelitis syndrome.

In the first month, chiropractic modalities, including therapeutic ultrasound therapy (three times a week) and myofascial mobilization ie, Graston ${ }^{\circledR}$ technique (one to two times a week), were utilized to release stiffness and restore function of the muscles and joints. In the following two months, spinal adjustments (diversified technique) and spinal traction were performed two times a week to retrain posture and body mechanics in order to reduce the pain during activities of daily living and work.

The patient noticed an increase in leg strength; muscle pain and cramps were relieved during the course of treatment. Reassessment at 3-month treatment completion, her surface EMG with reduced hyperactivity at most vertebral segments was indicative of improved paraspinal neuromuscular functions. She continued the treatment twice a month for further 3 months. At the reassessment at six months of treatment, self-reported improvements were recorded in all four domains of the WHOQOL-BREF. ${ }^{7}$ The scores were reported as $44 \%$ (from $31 \%$ ) in physical health, $88 \%$ (from $44 \%$ ) in psychological health, $75 \%$ (from $50 \%$ ) in social relationships, and $88 \%$ (from $44 \%$ ) environmental health.

\section{Discussion}

During the post-polio eradication era, the rate of isolation of nonpolio enteroviruses remains a clinical yardstick for the surveillance of acute flaccid paralysis (AFP) cases in the field. Poliomyelitis and AFP are illnesses that cause sudden weakness and floppy paralysis in children. Comprehensive AFP surveillance, which is essential for global certification of polio eradication, includes certification, containment, integration and eradication. ${ }^{8}$ Today, 179 of 194 the World Health Organization (WHO) member states conduct AFP surveillance and submit weekly AFP reports to WHO regional offices and WHO headquarters. In 2018, thirty-three wild PV cases were reported in endemic countries (21 in Afghanistan, 12 in Pakistan, and 0 in Nigeria). Seventy vaccine-derived PV cases occurred in non-endemic countries (26 in Papua New Guinea, 20 in the Democratic Republic of Congo, 13 in Somalia, 10 in Niger, and 1 in Mozambique). Other 34 vaccine-derived PV cases occurred in endemic countries (all 34 in Nigeria). The incidence of non-polio AFP in Afghanistan, Pakistan and Nigeria were 3378, 12256 and 9047 cases, respectively; corresponding to an AFP rate of 20.68/100,000, 20.01/100,000 and 12.88/100,000 children, respectively. Internationally, the incidence of non-polio AFP is $1-2 / 100,000$ children $<15$ years old. ${ }^{9}$ The Global Polio Eradication Initiative (GPEI) Strategic Plan 2019-2023 lays out the roadmap to achieving and sustaining a world free of all polioviruses [WHO]. ${ }^{8}$

Post-polio syndrome (PPS), a sub-category of the late effects of polio, is a neurologic disorder characterized by new weakness, muscle fatigability, general fatigue and muscle and joint pain in polio survivors. ${ }^{5}$ The most possible pathophysiology of new weakness is related to the exhaustion of the motor units (reinnervation mechanisms) that formed after the attack. ${ }^{1,10}$ Premature ageing of the surviving neurons may occur due to having been weakened by the previous episode and long-term compensation of the orphaned muscle fibers for the denervated neurons. ${ }^{2}$ Muscle pain is very common and is thought to be due to overuse of weak muscles or of other muscle groups which are compensating for the weakened polio-affected muscles. ${ }^{3}$ Joint pain can also be significant, typically caused by abnormal biomechanics, leg length inequality, muscle atrophy, joint stiffness, and age-relative changes. ${ }^{3}$ Some survivors have neuropathic pain, mainly caused by nerve impingement or disc herniation. 
While the search for a viral cause continues, there is no conclusive evidence to support that PPS is due to reinfection with the poliovirus or an immune-mediated condition. ${ }^{1}$ Emphasis is given to the possible pathogenic role of a persistent PV infection ${ }^{11}$ and chronic inflammation. ${ }^{1}$ A number of in vitro models showing persisting viruses in cell cultures have been reported. ${ }^{1}$ Experimental studies have also shown that PV may cause persistent infection and paralysis upon immunosuppression in mice. ${ }^{12}$ Immunodeficient persons are predisposed to a chronic PV infection, not only upon viral infection, but also following vaccination with attenuated virus. ${ }^{1}$ Persistent infection may be associated with the upregulation of pro-inflammatory cytokines and chemokines. ${ }^{13}$ Persistent infections likely contribute to PPS in the host.

The diagnosis of PPS is an exclusion diagnosis with no specific test or analysis, and the role of the investigation is to rule out every other possible cause for the new symptoms and clinical deterioration. ${ }^{6}$ The EFNS (European Federation of Neurological Societies) criteria ${ }^{3}$ seem to be universally accepted. The criteria are as follows: 1) Confirmed history of acute paralytic polio with signs of denervation on electromyogram. 2) Partial or complete recovery from acute paralytic poliomyelitis, followed by at least 15 years of stable neurologic function. 3) Gradual or sudden onset of progressive and persistent muscle weakness or abnormal muscle fatigability. 4) Symptoms persist for at least 1 year. 5) Exclusion of other neuromuscular, medical, and orthopedic conditions as causes of the problems. ${ }^{3}$

Interdisciplinary care is the best strategy for patients with PPS. Conservative treatment is a cornerstone of management of PPS. Tailored rehabilitation including muscular strengthening, aquatic therapy and endurance training have shown to have significant benefit on muscular exertion, endurance and pain relief. ${ }^{14} \mathrm{~A}$ variety of medications (such as acetaminophen, benzodiazepams and narcotics) are used to treat postpolio muscle pain, but with little effect. The use of tricyclic antidepressants, especially amitriptyline, can help with pain and also fatigue. Properly fitted orthoses can improve the biomechanical movement pattern and be energy-saving. ${ }^{3}$ Addressing postural deformities can often be helpful in minimizing or preventing biomechanical pain and strengthening muscles for sitting, standing, walking and other activities. Orthopedic surgery is occasionally recommended in the treatment of polio survivors. The goals of surgery include pain relief, deformity correction, muscle transfers to counter muscle force imbalance, stabilizing unstable joints, and allowing for more effective use of orthoses and braces. ${ }^{15}$

\section{Conclusion}

Post-polio syndrome is related to the exhaustion of the motor units that form decades after the polio attack. This case report describes the effectiveness of manual interventions in assisting our patient in restoring the level of function and alleviating pain. The limitation of the current report is that it is just a single case. Further comparison with more existing therapeutic regimens is warranted to clarify these issues.

\section{Ethics, consent and permissions}

Written informed consent has been provided by the patient to have the case details published. Ethics approval is not required for a retrospective chart review of anonymous patient data. No institution approval was required to publish the case details.

\section{Author contributions}

Both authors contributed to data analysis, drafting or revising the article, gave final approval of the version to be published, and agree to be accountable for all aspects of the work.

\section{Disclosure}

The authors report no conflicts of interest in this work.

\section{References}

1. Baj A, Colombo M, Headley JL, et al. Post-poliomyelitis syndrome as a possible viral disease. Int $J$ Infect Dis. 2015;35:107-116. doi:10.1016/j.ijid.2015.04.018

2. Groce NE, Banks LM, Stein MA. Surviving polio in a post-polio word. Soc Sci Med. 2014;107:171-178. doi:10.1016/j. socscimed.2014.02.024

3. Farbu E, Gilhus NE, Barnes MP, et al. EFNS guideline on diagnosis and management of post-polio syndrome. Report of an EFNS task force. Eur $J$ Neurol. 2006;13(8):795-801. doi:10.1111/j.14681331.2006.01385.x

4. Halstead LS, Rossi CD. New problems in old polio patients: results of a survey of 539 polio survivors. Orthopedics. 1985;8(7):845-850.

5. Trojan DA, Cashman NR. Post-poliomyelitis syndrome. Muscle Nerve. 2005;31(1):6-19. doi:10.1002/mus.20259

6. Farbu E, Gilhus NE, Barnes MP, et al. Post-polio syndrome. In: Gilhus NE, Barnes MP, Brainin M, editors. European Handbook of Neurological Management. Vol. 1. 2nded. Oxford: Blackwell Publishing; 2011:311-319.

7. Almeida-Brasi CC, Silveira MR, Silva KR, et al. Quality of life and associated characteristics: application of WHOQOL-BREF in the context of primary health care. Cien Saude Colet. 2017;22(5):1705-1716. doi:10.1590/1413-81232017225.20362015

8. World Health Organization. The GPEI Polio Endgame Strategy 20192023. Geneva: WHO; 2019. Available from: http://polioeradication. org/wp-content/uploads/2019/06/english-polio-endgame-strategy.pdf. Accessed July 31, 2019.

9. World Health Organization. The Polio Eradication and Endgame Strategies. Geneva: WHO Available from: https://extranet.who.int/ polis/public/CaseCount.aspx. Accessed July 31, 2019. 
10. Emeryk B, Rowińska-Marcińska K, Ryniewicz B, HausmanowaPetrusewicz I. Disintegration of the motor unit in post-polio syndrome. Part II. Electrophysiological findings in patients with post-polio syndrome. Electromyogr Clin Neurophysiol. 1990;30:451-458.

11. Julien J, Leparc-Goffart I, Lina B, et al. Postpolio syndrome: poliovirus persistence is involved in the pathogenesis. J Neurol. 1999;246:472-476.

12. Jubelt B, Meagher JB. Poliovirus infection of cyclophosphamidetreated mice results in persistence and late paralysis: II. Virologic studies. Neurology. 1984;34:494-499. doi:10.1212/wnl.34.4.494
13. Gonzalez H, Khademi M, Borg K, Olsson T. Intravenous immunoglobulin treatment of the post-polio syndrome: sustained effects on quality of life variables and cytokine expression after one year follow up. $J$ Neuroinflammation. 2012;9:167-178. doi:10.1186/1742-2094-9-167

14. Tiffreau V, Rappin A, Serafi R, et al. Post-polio syndrome and rehabilitation. Ann Phys Rehabil Med. 2010;53(1):42-50. doi:10.1016/j.rehab.2009.11.007

15. Sheth NP, Keenan MAE. Orthopedic surgery considerations in postpolio syndrome. Am J Orthop. 2007;36(7):348-353.

\section{Publish your work in this journal}

The International Medical Case Reports Journal is an international, peer-reviewed open-access journal publishing original case reports from all medical specialties. Previously unpublished medical posters are also accepted relating to any area of clinical or preclinical science. Submissions should not normally exceed 2,000 words or 4 published pages including figures, diagrams and references. The manuscript management system is completely online and includes a very quick and fair peer-review system, which is all easy to use. Visit http://www.dovepress.com/testimonials.php to read real quotes from published authors. 